\title{
ESTUDOS TAXONÔMICOS EM PIPERACEAE DO SEMIÁRIDO, COM ÊNFASE NA CHAPADA DIAMANTINA
}

\author{
Andrezza Lóren de Góes Nascimento ${ }^{1}$ Reyjane Patrícia Oliveira $^{2}$, Hemilly \\ Marques Seixas $^{3}$ \\ 1. Bolsista PIBIC/CNPq, Graduanda em Bacharelado em Ciências Biológicas, Universidade Estadual de Feira \\ de Santana, e-mail: andrezzaloren@gmail.com \\ 2. Orientadora, Departamento de Ciências Biológicas, Universidade Estadual de Feira de Santana, e-mail: \\ rpatricia@uefs.br \\ 3. Graduanda em Bacharelado em Ciências Biológicas, Universidade Estadual de Feira de Santana, e-mail: \\ hemillyseixas@hotmail.com
}

PALAVRAS-CHAVE: Florística; Semiárido; Piperales.

\section{INTRODUÇÃO}

O Semiárido brasileiro corresponde basicamente à delimitação do bioma Caatinga e está praticamente incluído na região Nordeste, juntamente com o norte de Minas Gerais, na região Sudeste, correspondendo a mais de $10 \%$ do território Nacional (Moura \& Campos 2004). A vegetação do Semiárido oferece diversos benefícios ambientais, econômicos e sociais, e muitos inventários já foram realizados, especialmente ao longo da Chapada Diamantina, que representa um mosaico vegetação nessa região, tendo o campo rupestre como o principal tipo vegetacional (Giulietti \& Queiroz 2006).

Apesar de toda a diversidade já detectada, alguns grupos de plantas permanecem pouco estudados na região, como é o caso de Piperaceae, representante da ordem Piperales (Judd et al. 2009). Essa família inclui cinco gêneros e 3.700 espécies (Christenhusz, et al. 2017), tendo grande riqueza na flora do Brasil, incluindo quatro gêneros e ca. 500 espécies (Guimarães et al. 2015), ocorrentes especialmente em áreas florestais. Porém, são escassos os taxonomistas da família no país, com muitas amostras da Bahia sem identificação nas coleções de herbário.

O presente estudo visou contribuir para o conhecimento atualizado da composição florística do Semiárido, com base no levantamento da família Piperaceae na Chapada Diamantina, contribuindo também para o conhecimento da família no Brasil. O trabalho apresenta um checklist atualizado das espécies dessa família na região, com chave e comentários para os gêneros, assim como indicação de espécies endêmicas nos mesmos, evidenciando também novos registros de ocorrência para a Bahia.

\section{MATERIAL E MÉTODOS OU METODOLOGIA}

A metodologia para esse plano de trabalho seguiu os parâmetros gerais adotados em estudos florísticos e taxonômicos, especialmente quanto às áreas prioritárias para coletas, definidas pela rede PPBIO Semiárido. Inicialmente, os limites da Chapada Diamantina foram analisados através da definição da SEi (2018), o qual divide a Bahia em 27 Territórios de Identidade, estando os municípios da Chapada Diamantina inseridos primordialmente em três deles (Território da Chapada Diamantina, o Território do Piemonte do Paraguaçu e o Território do Piemonte da Diamantina), incluindo 46 municípios. 
Foram realizadas coletas ao longo dessa região, utilizando procedimentos habituais que envolvendo prensagem, secagem e confecções de exsicatas (Mori et al. 1989). Os espécimes coletados foram analisados com a ajuda da literatura disponível (Judd et al. 2009; Souza \& Lorenzi 2008; Yuncker 1972, 1973, 1974) e incorporados na coleção do herbário da Universidade Estadual de Feira de Santana (HUEFS, segundo Thiers 2018). Todo o levantamento sobre amostras da família Piperaceae na Chapada Diamantina depositadas em outros herbários, incluindo tipos nomenclaturais, foi feito através de consultas a herbários virtuais nas bases de dados do Species link e Reflora, entre outros. Os dados obtidos foram devidamente triados para uma atualização e correção de nomes com auxílio da plataforma da Flora do Brasil 2020 (http://floradobrasil.jbrj.gov.br) e da base de dados do Missouri Botanical Garden (www.tropicos.org). Literatura especializada com informações sobre a vegetação da região foi utilizada para a indicação de endemismos (Giulietti et al., 2002, Flora do Brasil 2020).

\section{RESULTADOS E/OU DISCUSSÃO (ou Análise e discussão dos resultados)}

As buscas realizadas inicialmente indicavam a presença de 751 espécimes, pertencentes a 46 espécies de Piperaceae para a extensão da Chapada Diamantina, representantes dos gêneros Peperomia Ruiz \& Pav. e Piper L., que estão inseridos na subfamília Piperoideae, a família ainda possui outras duas subfamílias: Verhuellioideae e Zippelioideae. Após estudos morfológicos detalhados nesses gêneros, uso de chaves de identificação para as espécies e atualizações nomenclaturais, o número de espécies na região foi reduzido. É aqui confirmada a ocorrência de 42 espécies, sendo 23 de Peperomia [P. alata Ruiz \& Pav., P. blanda (Jacq.) Kunth, P. campinasana C.DC., $P$. corcovadensis Gardner, P. crinicaulis C.DC., P. diamantinensis Carv-Silva, E. F. Guim $\&$ PEAS Câmara, $P$. diaphanoides Dahlst., $P$. decipiens C.DC., $P$. galioides Kunth, $P$. glabella (Sw.) A.Dietr, P. glazioui C.DC., P. hernandiifolia (Vahl) A.Dietr, P. nitida Dahlst, P. obtusifolia (L.) A.Dietr, P. oreophila Henschen, P. pseudoestrellensis C.DC. $P$. rotundifolia (L.) Kunth, $P$. sincorana C.DC., P. stenocarpa Regel, $P$. subrubricaulis C.DC., P. subrubrispica C.DC., P. tetraphylla Hook. \& Arn., P. urocarpa Fisch. \& C.A. Mey] e 19 pertencentes a Piper [P. aduncum L., P. amalago L., P. arboreum Aubl., P. bennettianum C.DC., $P$. caldense C.DC., $P$. cernuum Vell., $P$. chimonanthifolium Kunth, $P$. crassinervium Kunth, $P$. cuyabanum C.DC., $P$. dilatatum Rich., P. flavicans C.DC., P. fuligineum Kunth, P. hispidinervum C.DC., P. hispidum Sw., $P$. lhotzkyanum Kunth, $P$. macedoi Yunck, $P$. richardiifolium Kunth, $P$. tectoniifolium Kunth e P. umbellatum L.].

Na coleção do HUEFS estão depositados 554 espécimes de Piperaceae coletadas na Bahia, dos quais 159 pertencem a municípios inseridos na Chapada Diamantina (Fig. 1), distribuídos em 27 espécies. Os quatro nomes citados no Species link e descartados do presente trabalho são: Peperomia augescens Miq., Peperomia quadrifolia (L.) Kunth, Peperomia stroemfeltii Dahlst e Piper subsilvestre C.DC, e a exclusão dessas espécies da lista se deu porque se tratava de registros duvidosos ou incorretos. As mesmas não estavam formalmente registradas para a Bahia, algumas estavam registradas como cf., com locais de ocorrência muito discrepantes, mas principalmente, porque suas características morfológicas não eram compatíveis com as descrições dos materiais tipo dessas espécies nem com as chaves de identificação consultadas. 
Dentre as 42 espécies confirmadas, 19 são exclusivas do Brasil. Peperomia foi o gênero com maior riqueza na Chapada Diamantina, tendo duas espécies endêmicas da Chapada Diamantina ( $P$. sincorana e $P$. diamantinensis, sendo esta primeira ocorrente também em municípios adjacentes; Tab. 1). E ainda, Peperomia diaphanoides representa uma nova ocorrência para o estado da Bahia.

Dentre os municípios da Chapada, o maior número de espécies foi detectado para Lencóis (22 espécies), estando também bem representada em Abaíra (16), Palmeiras (15) e Rio de Contas (13) (Fig. 1).

Tabela 1. Espécies de Piperaceae ocorrentes na Chapada Diamantina e endêmicas do Brasil, da Bahia e da Chapada, de acordo com Guimarães (et al. 2015).

\begin{tabular}{llll}
\hline Espécie & Brasil & Bahia & Chapada \\
\hline Peperomia campinasana C.DC. & $\mathrm{X}$ & & \\
Peperomia crinicaulis C.DC. & $\mathrm{X}$ & & \\
Peperomia decipiens C.DC. & $\mathrm{X}$ & & \\
Peperomia diamantinensis Carv-Silva, E. F. Guim \& & $\mathrm{X}$ & $\mathrm{X}$ & $\mathrm{X}$ \\
PEAS Câmara & & & \\
Peperomia glazioui C.DC. & $\mathrm{X}$ & & \\
Peperomia oreophila Henschen & $\mathrm{X}$ & & \\
Peperomia pseudoestrellensis C.DC. & $\mathrm{X}$ & & \\
Peperomia sincorana C.DC. & $\mathrm{X}$ & $\mathrm{X}$ \\
Peperomia stenocarpa Regel & $\mathrm{X}$ & & \\
Peperomia subrubricaulis C.DC. & $\mathrm{X}$ & & \\
Peperomia subrubrispica C.DC. & $\mathrm{X}$ & & \\
Piper bennettianum C.DC. & $\mathrm{X}$ & & \\
Piper caldense C.DC. & $\mathrm{X}$ & & \\
Piper chimonanthifolium Kunth & $\mathrm{X}$ & & \\
Piper flavicans C.DC. & $\mathrm{X}$ & \\
Piper hispidinervum C.DC. & $\mathrm{X}$ & \\
Piper macedoi Yunck & $\mathrm{X}$ & \\
Piper richardiifolium Kunth & $\mathrm{X}$ & \\
Piper tectoniifolium Kunth & $\mathrm{X}$ & \\
\hline
\end{tabular}
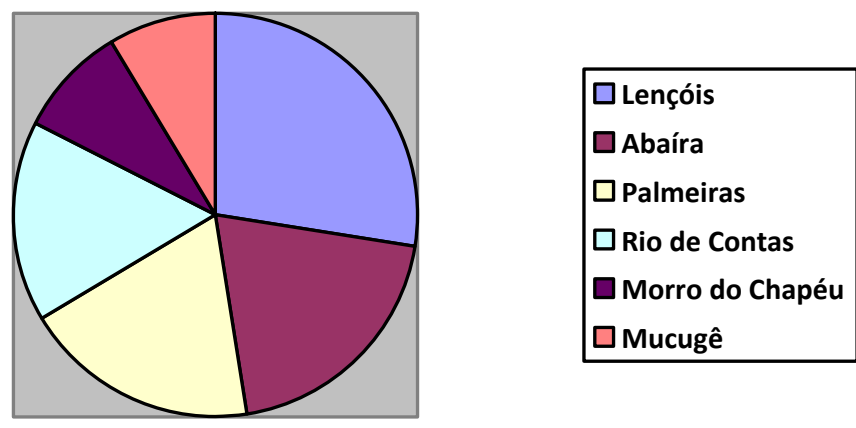

Figura 1: Munícipios da Chapada Diamantina com maior riqueza de Piperaceae. 


\section{CONSIDERAÇÕES FINAIS}

A partir das análises realizadas foi possível confirmar a ocorrência de 42 espécies de Piperaceae na Chapada Diamantina, representantes dos gêneros Peperomia e Piper. Todos os materiais da família depositados no herbário HUEFS foram analisados, além de vários outros acessados através de herbários virtuais, incluindo tipos nomenclaturais. Peperomia apresentou maior riqueza, incluindo duas espécies endêmicas da Bahia, uma delas exclusiva da Chapada e uma nova ocorrência para o Estado. O município com maior riqueza foi Lençóis (22 espécies, dos dois gêneros) e todas essas informações são de grande importância para subsidiar estudos posteriores sobre a flora da Chapada e sobre Piperaceae, incluindo estudos aplicados com essa família que merece destaque pelo grande potencial que detém, especialmente taxonômico, evolutivo e fitoquímico.

\section{REFERÊNCIAS}

CHRISTENHUSZ, M. J. M.; FAY, M. F. \& CHASE, M. W. 2017. Plants of the world: an illustrated encyclopedia of vascular plants. The University of Chicago Press, ISBN 9780226536705. p. 99.

GIULIETTI, A. M. \& QUEIROZ, L. P. (Org.). 2006. Instituto do Milênio do Semiárido. 1. ed. Recife: APNE, 2000p.

GIULIETTI, A.M. ET AL. 2002. Espécies endêmicas da Caatinga. In: E.V.C.B. Sampaio et al. (Eds.) Vegetação \& Flora da Caatinga. Associação Plantas do Nordeste, CNIP, Recife.

GUIMARÃES, E.F.; CARVALHO-SILVA, M.; MONTEIRO, D.; MEDEIROS, E.S.; QUEIROZ, G.A. 2015. Piperaceae in Lista de Espécies da Flora do Brasil. Jardim Botânico do Rio de Janeiro. Disponível em: <http://floradobrasil.jbrj.gov.br/jabot/floradobrasil/FB190>. Acesso em: 25 Jul. 2018. JUDD, W. S.; CAMPBELL C. S.; KELLOGG E. A.; STEVENS P. S.; DONOGHUE M. J. 2009. Sistemática Vegetal: um enfoque filogenético. $3^{\mathrm{a}}$ Ed. Porto Alegre, Artemed.

MORI, S.A.; SILVA, L.A.M.; LISBOA, G. \& CORADIN, L. 1989. Manual de Manejo de Herbário Fanerogâmico. 2a ed. Ilhéus, Centro de Pesquisas do Cacau.

MOURA, A. S. \& CAMPOS, G. C. (COORD.) 2004. Cenários para o bioma Caatinga. Conselho Nacional da Reserva da Biosfera da Caatinga, Secretaria da Ciência, Tecnologia e Meio Ambiente de Pernambuco, Recife.

SOUZA, V. C.; LORENZI, H. 2008. Botânica sistemática: guia ilustrado para identificação das famílias de Angiospermas da flora brasileira, baseado em APG II. Nova Odessa, SP: Instituto Plantarum de Estudos da Flora.

YUNCKER, T.G. 1972. The Piperaceae of Brazil, I. Piper - Group I, II, III, IV. Hoehnea 2: 19-366.

YUNCKER, T.G. 1973. The Piperaceae of Brazil, II: Piper - Group V, Ottonia; Pothomorphe; Sarcorchachis. Hoehnea 3: 29-284.

YUNCKER, T.G. 1974. The Piperaceae of Brazil, III: Peperomia - taxa of incertain status. Hoehnea 4: 71-413. 